\title{
The Role of Experimental Test Beds for the Systems Testing of Future Marine Electrical Power Systems
}

\author{
M. H Syed*, E. Guillo-Sansano*, A. Avras ${ }^{\star}$, A. Downie ${ }^{\star}$, K. Jennet ${ }^{\star}$, G. M. Burt*, F. Coffele ${ }^{\star}$, A. Rudd ${ }^{\dagger}$ \\ and C. Bright ${ }^{\dagger}$ \\ ${ }^{*}$ Institute for Energy and Environment, University of Strathclyde, Glasgow, UK \\ ${ }^{\star}$ Power Networks Demonstration Centre, University of Strathclyde, Glasgow, UK \\ $\dagger$ Rolls-Royce, Derby, UK \\ mazheruddin.syed@strath.ac.uk, a.avras@strath.ac.uk and chris.bright@ rolls-royce.com
}

\begin{abstract}
Marine electrical power systems (MEPS) are experiencing a progressive change with increased electrification - incorporation of distributed power generation, high power density requirement, increased storage integration, availability of alternative technologies and incorporation of novel loads to name a few. In recent years, smart grid (advanced land based power systems) concepts have increasingly been incorporated within MEPS to leverage on their proven advantages. Due to the distinct nature of the two power systems, upon incorporation, the solutions need to be further proven by simulations and experimentation. This paper presents two smart grid enabled MEPS test beds at the University of Strathclyde developed to allow for proof of concept validations, prototyping, component characterization, test driven development/enhancement of emerging MEPS solutions, technologies and architectures. The capabilities of the test beds for rapid proof of concept validations and component characterization are discussed by means of two case studies. Drawing on from the two case studies, this paper further presents a discussion on the requirements of systems testing of future more electric MEPS.
\end{abstract}

Index Terms-experimental evaluation, marine electrical power systems, systems testing and test beds.

\section{INTRODUCTION}

Validation and testing in smart grids (advanced land based power systems) has aided the wide-scale acceptance and deployment of a number of novel technologies. Three categories of validation approaches can be identified for power systems domain as [1]: (i) simulation only methods [2], (ii) real-world based methods (experimental laboratories and field trials) [3] and (iii) a combination of virtual and real-world based methods [4]. Real-world based methods comprise laboratory validations and field trials, where the latter offers a higher degree of confidence but is expensive and limited to steady state validations; the former is less expensive and offers more flexibility in terms of validation under wider operating conditions. The capabilities and flexibility offered by experimental laboratories is extended by the incorporation of digital real time simulators (DRTS) enabling controller hardware-in-the-loop (CHIL) and power hardware-in-the-loop (PHIL) setups (i.e. a combination of virtual and real-world methods). In the recent past, the role of experimental laboratories in accelerating the development, deployment and acceptance of novel technologies (control solutions or equipment) has been evidenced. A recent example of which is the deployment of normally soft open points in the low-voltage networks in London by UK Power Networks [5].

The maturity of smart grid research and deployment in land based power systems has drawn the attention of marine electrical power systems (MEPS) and aero-electrical power system developers. In both of these domains a number of smart grid technologies and concepts are being considered for adoption due to their proven/demonstrated benefits in utility power systems. Although the benefits of incorporating smart grid concepts within MEPS can be speculated; proof of their performance is required before large scale adoption and deployment can be considered. Similarly to smart grid in the utility domain, experimental validation can play a key role in:

- the assessment of the impact of incorporating novel components such as high power dense direct current loads and their power electronic interfaces [6].

- evaluating the capability of novel smart coordinated control strategies [7].

- the assessment of novel MEPS architectures [8].

A number of test beds for MEPS validation have been reported in literature, a few examples of which are in [6][8]. Many industrial applications, including but not limited to hospitality, freight, and naval, have identified the value of such test beds. These test beds have already eliminated some of the crucial risks associated with pursuing simulation only validation or the direct deployment of new technologies. Pure simulations can lack the fidelity associated with testing real world equipment while direct deployment is limited by time and physical constraints of the system. In this paper, two questions worthy of discussion under the realm of MEPS validation and testing have been identified and dealt with:

- a number of infrastructures are available around the world, predominantly working with land based power systems. The capability and suitability of such infrastructures to be adapted for MEPS testing is discussed with examples.

- with the increasing complexity of MEPS, the concept of systems testing for rigorous validations is introduced and emphasized.

The paper is organized as follows. The established methods for land based power systems experimental evaluation, a 
comparison of its role in appraisal of novel technologies to the scale of technology readiness level (TRL) is drawn and the concept of systems testing is introduced in Section II. In Section III, the paper further shares the experience of adaptation of two experimental test beds, designed for testing of smart grids, for the validation and testing of MEPS. Their use is demonstrated by means of two use cases: (i) coordinated control of energy storage systems (ESS) for mitigation of frequency transients on MEPS and (ii) characterization of flywheel ESS (FESS) for use on MEPS. Section IV draws the insights on the future requirements of MEPS validations, where the need for a systems testing approach is highlighted while Section V concludes the paper.

\section{Experimental Evaluation of Electrical Power SYSTEMS}

\section{A. Established Methods for Experimental Evaluation of Power Systems}

Experimental evaluation of power system components, such as the characterization of Photo-Voltaic inverters or their conformance testing to a standard, are well understood and undertaken regularly [9]. The power systems have now evolved into a cyber-physical system, i.e. a smart grid, enabled by the integration of state-of-the-art automation and communications technologies [10]. This has further enabled the development of novel intelligent control solutions, the advantages of which have been brought forward by a number of research and demonstration projects [11]. However, this evolution has added a new level of complexity to the experimental evaluation of power systems while at the same time it has become a necessity that any novel intelligent control solution is experimentally evaluated to allow for paving its way forward into the market as a product or service. To help address this, in [12], a validation chain that comprises five phases to thoroughly evaluate novel intelligent control solutions has been proposed and is briefly discussed below:

- Phase I: First stage to validation is a pure software simulation approach wherein the power network and control are developed within one simulation platform. A plethora of commercially available simulation tools can be utilised, some designed for steady state analysis (for example PSSE [13], [14]) and some more specifically for transient analysis (for example PSCAD [15], [16]).

- Phase II: To adequately capture the interface between power and control systems, a natural progression is the use of two dedicated software simulation tools, one for power system and one for control system in closed loop configuration. In other words, phase II is a co-simulation or software-in-the-loop approach [17], [18].

- Phase III: The next phase involves testing of the control in a CHIL setup [19], [20]. The advantages offered by CHIL include: (i) the ability to reveal hidden issues of the control solution (often in terms of its implementation and real-time run capability) and (ii) allows for control solutions performance to be evaluated under realistic conditions.

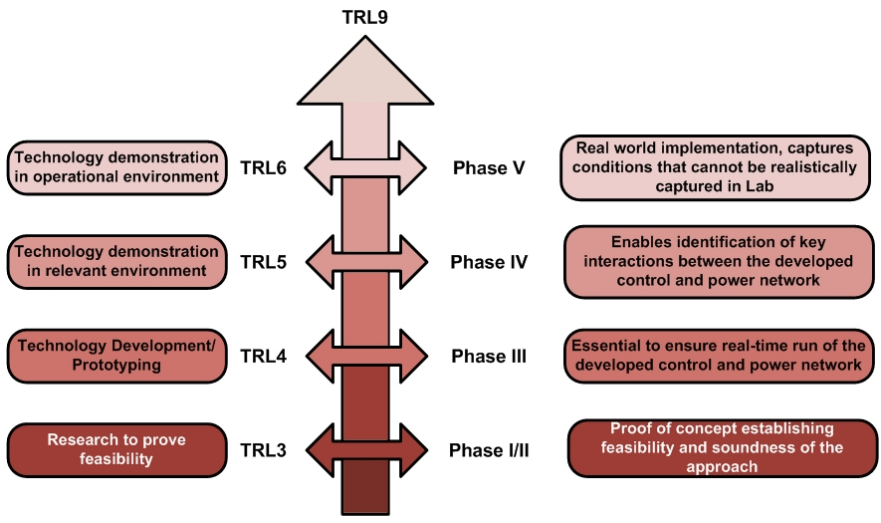

Fig. 1: A technology readiness level and validation chain comparison.

- Phase IV: The penultimate phase of the validation chain is the combination of CHIL and PHIL techniques in one simulation/experimental setup [21], [22]. Such a setup enables validation of controls under a wide range of scenarios, which can be difficult, risky, expensive, and even impossible to perform in real life systems.

- Phase V: The final phase of validation is by means of its implementation within the field.

\section{B. Mapping to Technology Readiness Level}

The validation chain presented above is intended to be carried out serially for rigorous validation of a control solution developed for power systems. It is important to note that each phase of the validation chain offers significant advances in terms of the readiness of the proposed control solution. To illustrate this better, consider the relation drawn between each phase of the validation chain and technology readiness level (TRL) [23] in Fig. 1. Phase I and II effectively establish the feasibility and soundness of the developed control, providing a proof of concept, enhancing the control to TRL 3. Following a successful proof of concept, the implementation of the developed control on a hardware controller board in phase III, capable of running in real-time, offers validation with increased fidelity. This can be related to the control achieving TRL 4. The combined CHIL and PHIL experimental setup of phase IV offers validation with highest fidelity, capturing interactions between integrated control and power components, appraising the control to TRL 5. Field trials can be viewed as demonstration of the developed control in an operational environment and hence mapped to TRL 6 .

\section{Systems Testing}

Systems testing or systems level testing is referred to the testing of a power component or control as part of a representative power system. Similarly, a subsystem could be also tested as part of a larger power system. In other words, systems testing refers to the rigorous evaluation under most representable real-world characteristics possible at scale. There are two main characteristics of systems testing identified that distinguish it from the existing methods to power systems evaluation: 
- As mentioned earlier, the power system has evolved into a cyber-physical system, where a number of domains have been integrated to facilitate its transition and improved operation. Examples of such domain integration include the communications domain and the metrology domain. The incorporation of these domains for the experimental evaluation is the enabler to systems level testing. For example, the use of appropriate communications protocols and communications emulation within Phase V of validation chain allows for real-world characteristics to be incorporated. In a similar manner, the incorporation of novel power systems metrology equipment such as the phasor measurement units in a distributed manner where required rather than its substitution by conventional measurements available by a centralized SCADA of respective laboratories.

- Compared to the conventional PHIL setups where typically a single component is connected to a Digital Real-Time Simulator (DRTS) while the remainder of the power system is simulated in real-time, systems testing is envisioned to push the boundaries of such experimental setups. The presence of a larger system, i.e., a group of components or an area of power system (as in [24]), allows for an appropriate implementation and evaluation of control schemes and provides a higher level of confidence in its performance.

\section{ExPERimental EVAluation of MARine EleCtRICAL POWER SYSTEMS}

As has been highlighted in Section I, there is an increasing interest in the incorporation of smart grid concepts within MEPS to leverage on their proven advantages. In this subsection, the capability of existing research infrastructures, designed and developed for land based power system experimental evaluation, for the experimental evaluation of MEPS is discussed. First, the test bed infrastructures at University of Strathclyde are briefly presented followed by case studies where its adaptation and utilization for experimental evaluation of MEPS is demonstrated.

\section{A. Test Beds at University of Strathclyde}

The University of Strathclyde has two research infrastructures developed as test beds for power systems experimental evaluation and this subsection presents a brief description of the two.

1) Dynamic Power Systems Laboratory: The dynamic power systems laboratory (DPSL) is a $115 \mathrm{kVA}, 400 \mathrm{~V}$ three phase facility. The simplified one line diagram of of DPSL is shown in Fig. 2. As can be observed, the network is designed such that it can be split into 3 separate power islands (referred to as cells) under independent control (purple, green and orange), or brought together in any combination as a single system. This provides a high degree of flexibility enabling many different land-based or marine scenarios to be created.

The power network is further complemented by a digital real-time simulator from RTDS Technologies, allowing for

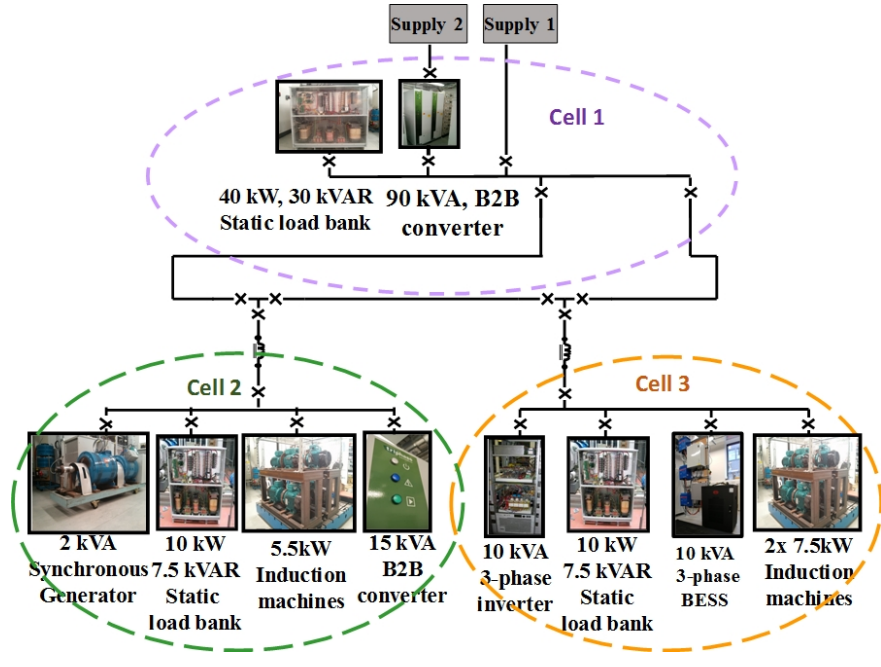

Fig. 2: Simplified one line diagram of dynamic power systems laboratory

CHIL and PHIL setups to be realized in conjunction with the 90kVA back-to-back (B2B) converter. Each cell of the network has a PMU installed with additional 64 PMUs available through the wide area monitoring, protection and control platform [25]. For the purpose of conducting scalable CHIL implementations, the facility provides up to 64 distributed controllers within its distributed controller platform, an example application of which can be found in [26]. The laboratory further supports all industry relevant communications protocols and incorporates two comunications emulators: (i) an NS-3 based communications emulator and (ii) a distributed communications emulator platform [27].

2) Power Networks Demonstration Centre: The Power Networks Demonstration Centre (PNDC) is a MW level industry facing facility hosting an $11 \mathrm{kV}$ and $400 \mathrm{~V}$ network environment representative of UK networks, providing a stable and configurable testing environment for different devices and smart technologies. Secure test bays can host pre-commercial testing of primary and secondary equipment. The motorgenerator allows for testing of islanding scenarios with multiple connection points for ESS and converter technologies. Furthermore, the real-time simulation capabilities and expertise of PNDC facilitate the design, testing and validation of new forms of generation, network components, demand-side

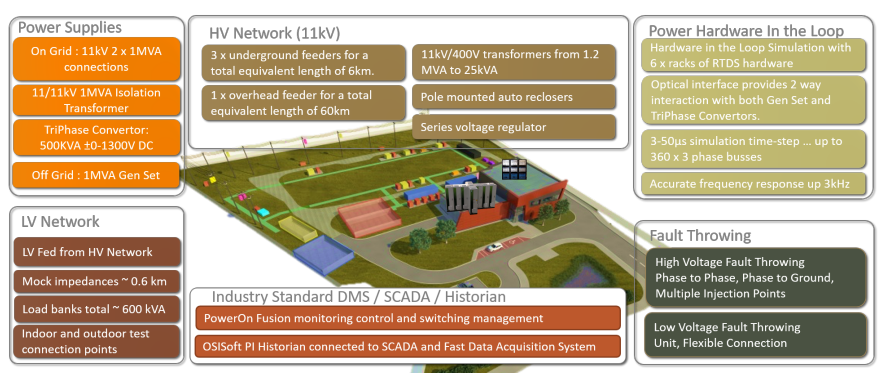

Fig. 3: PNDC capabilites and assets. 
management and storage systems. Finally, a Power (Amplifier) Interface makes possible the formation of HIL architectures in local and distributed fashion. The platform is domain agnostic and has been used for studies relating to utility, transport and naval applications.

\section{B. DPSL Case Study: Distributed ESS for Frequency Tran- sients Mitigation}

In this subsection, the capability of DPSL for rapid prototyping and proof of concept validation for the integration of novel technologies and its coordinated control is presented. The key objective is to analyze the integration of novel technologies (ESS in this case) into conventional MEPS and to evaluate its performance in mitigation of frequency transients. For this purpose, modular distributed ESS have been incorporated within the MEPS and are complemented with smart grid control functions for its coordinated response. The following subsections present the experimental setup, controls integration, the scenario and a discussion of the results.

1) PHIL Setup: The PHIL setup of the MEPS is shown in Fig. 4. As can be observed. the high voltage side of the network including the two step-down transformers are simulated within the DRTS with the low voltage side being represented by the equipment of DPSL. To ensure stability of the setup and to be able to adequately represent a portion of the MEPS network, the currents are scaled by a factor of $k=25$. The voltage of the point of common coupling (PCC) from the DRTS is sent to the $90 \mathrm{kVA}$ power interface for reproduction within the laboratory, while the currents measured in response to the voltage are sent back to the DRTS. To ensure accuracy of the PHIL setup, the time delay introduced due to the communication of voltages and currents to-and-from DRTS is compensated by sliding discrete Fourier transform based phase compensation technique presented in [28]. The process of initialization and synchronization of the PHIL setups is performed as described in [24].

2) MEPS Configurations: Two different system configurations, one representing MEPS of today and one representing a more electrically evolved MEPS with the addition of energy storage and its integrated control are utilized as described below:

- Conventional Configuration: This configuration is representative of existing MEPS where the incorporation of ESS is limited (non-existent or sometimes for emergency purposes only). The MEPS is powered by two 2MVA synchronous generators and no ESS is considered.

- Alternative Configuration: The alternative configuration is representative of a transitionary MEPS. Two different ESS technologies have been incorporated, i.e., the battery ESS (BESS) and the super-capacitor ESS. In order to offset the weight of integrating ESS as well as the increased capacity of the power system, the ratings of the generators are reduced to half the rating used for the conventional configuration (smaller generators supplemented with ESS). This allows for a more fair comparison of the two configurations.
3) Integrated Controls: In this subsection, the controls integrated within the MEPS are described. First the controls common within the two configurations are described, followed by the conventional configuration and alternative configuration respectively.

- Common Controls: To protect the MEPS under severe transients that result in frequency deviations that violate a defined threshold of safe operation, an under frequency load shedding is implemented with the threshold chosen as $4 \%$ below the nominal frequency.

- Conventional configuration controls: Within the conventional configuration, the generators operate with a droop of $13 \%$ for primary frequency regulation and are solely responsible for frequency restoration, i.e., secondary frequency control. Any disturbances within the MEPS are dealt with by the generators.

- Alternative configuration controls: The controls for alternative configurations are developed with two key objectives:(i) to minimize the frequency deviations and (ii) to reduce the duty on the two conventional generators, i.e., the generators are to experience little to no operational change during the transients. For this purpose, the generators operate with a very high droop (which translates into an almost non existent contribution to primary frequency regulation) while the modular BESS operates with a droop of $1 \%$ for primary frequency regulation. Both BESS and generators participate in secondary frequency control with a PI control gain ratio of 30:1, i.e., the BESS responds to the deviation in frequency 30 times faster than the generators. This prevents the generators from responding to any sudden changes in network conditions while at the same time ramping up slowly to contribute to frequency restoration. In addition, to allow the generators to operate at the highest efficiency (i.e. operate at rated power) an additional balance control loop is incorporated. It is assumed that additional measurements and communications infrastructure is incorporated within the MEPS that allows for real-time situational awareness to the generators balance control loop. The balance control loop operates much slower preventing excessive stress on the generators.

4) Scenario under Consideration: This scenario is developed to investigate the performance of the two configurations under the more common transients that occur on a MEPS during its day to day operations such as the accelerating and decelerating of the propulsion motors(PMs) at different speeds and the connection or disconnection of loads. Four cases are

TABLE I: Scenario I cases under consideration

\begin{tabular}{ll}
\hline \hline Case & Details \\
\hline \hline Case A & Starboard Side PM ramped from 0 to 1pu torque over 15s \\
Case B & Starboard Side PM ramped from 1 to 0pu torque over 15s \\
Case C & Starboard Side PM raised from 0 to 1pu torque instantaneously \\
Case D & Starboard Side PM lowered from 1 to 0pu torque instantaneously \\
\hline \hline
\end{tabular}




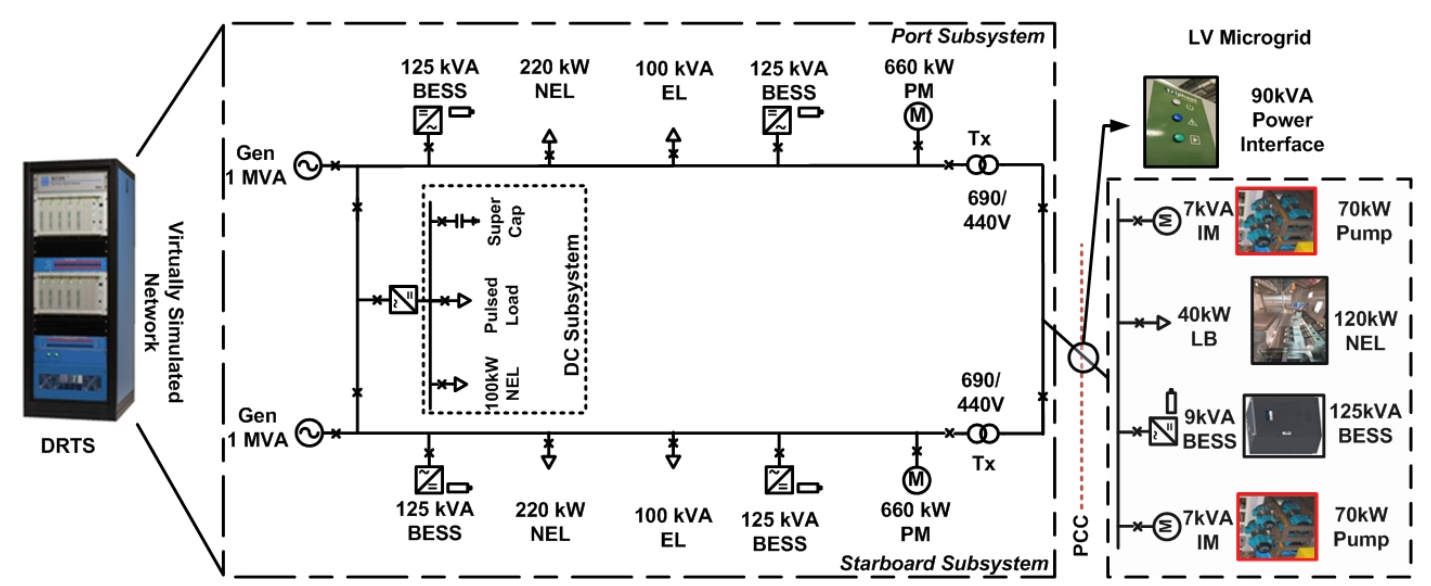

Fig. 4: PHIL setup utilized for MEPS experimental evaluation at DPSL.

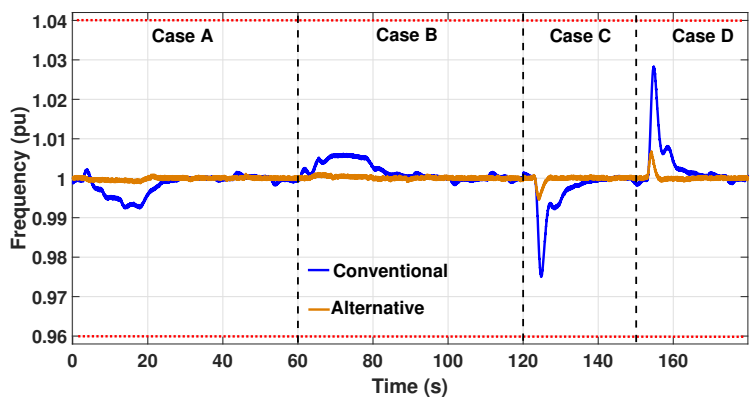

(a) System frequency.

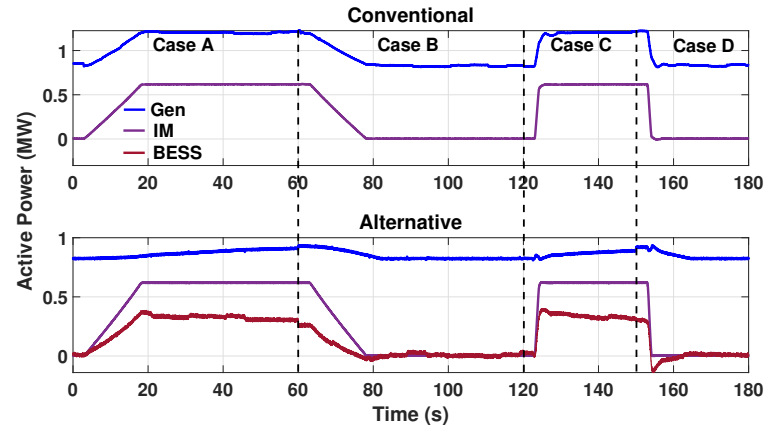

(b) Assets active power output.

Fig. 5: System response for scenario under consideration

considered within the scenario, as presented in Table I. The first two cases are representative of the vessel accelerating and decelerating while the latter two cases represent situations where a large load is suddenly switched on or off.

The system frequency response is presented in Fig. 5a while the assets active power output is presented in Fig. $5 \mathrm{~b}$. In the conventional configuration, any change in active power demand is covered by the generators as is evident from the results while deviations in frequency up to $0.008 \mathrm{pu}$ for Case A/B and up to 0.028pu for Case C/D is observed. To avoid large deviations in frequency, it is usually preferred to ramp PMs over a period of time. However, as can be observed from the results, with the incorporation of BESS the deviation in frequency with instantaneous raising/lowering of PMs is minimal. As can also be observed, a smoother frequency response is achieved with BESS. Furthermore, the performance of the additional balance control loop within the alternative configuration can be observed from Fig. 5b, where the generators slowly increase their power output up to $0.95 \mathrm{pu}$ (chosen as rated power). In most cases the generators of MEPS are oversized to deal with any load variation of the network, irrespective of the duration of peak loading and for redundancy purposes. As can be observed, in conventional scenarios with 2MVA generators, the generators only operate at $50 \%$ of their rated power.
5) Discussion: As is evident from the case study presented, the existing test bed was successfully utilized for rapid proof of concept validation. This paper only presents one scenario out of the many undertaken at the facility as the objective is to emphasize the role the existing test beds can play for supporting the transition of MEPS. Such practical rapid proof of concept demonstrations using test beds, that incorporate elements of real-world scenario, play an important role to convince potential stakeholders on the feasibility and capability of novel technologies and control over purely theoretical proof and analysis. Upon evolution of the developed controls with complex novel control solutions, following the validation chain, the test bed allows for the development of its prototype and its appraisal to TRL 5.

\section{PNDC Case Study: FESS Characterization}

In this subsection, the capability of PNDC for supporting the acceleration of novel technology development and deployment for MEPS applications is presented. The objective of the study is to characterize the response of a commercial flywheel energy storage system developed for MEPS. The following subsections present the experimental setup, control integration, the tests undertaken and discuss the results.

1) PHIL Setup: The setup utilized for testing of a $3.5 \mathrm{MJ}$ FESS is shown in Fig. 6a. The key components of the test 


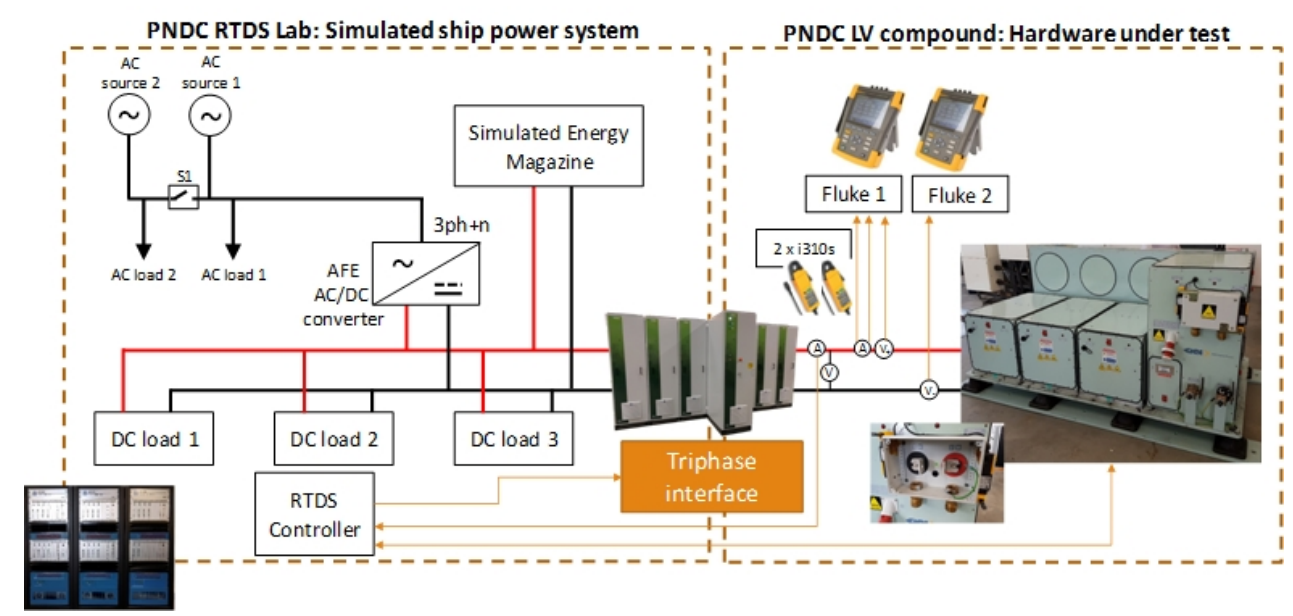

(a) PHIL setup utilized for MEPS experimental evaluation at PNDC.

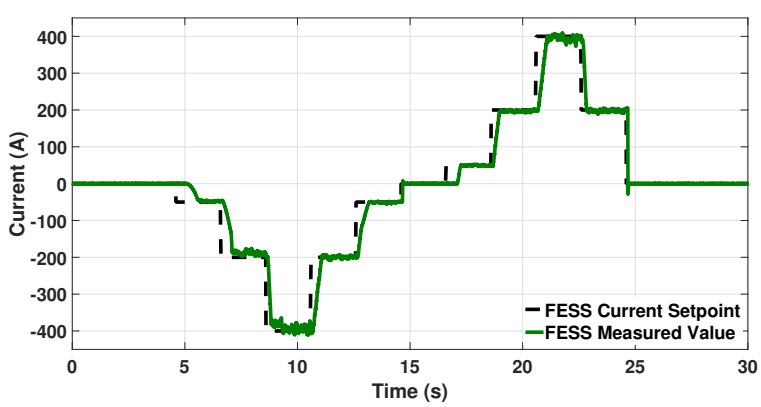

(b) Step change test response.

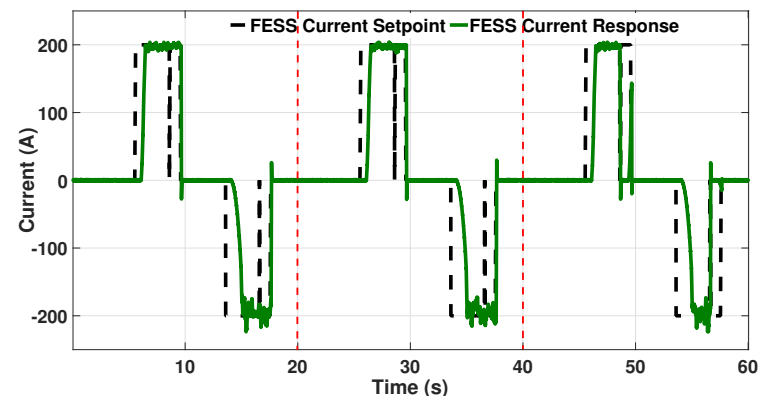

(c) Dropout test responses.

Fig. 6: Case Study 2 PHIL setup and results

platform are briefly summarized below:

- DRTS: DRTS is used for modelling the notional ship power system model. The DRTS also hosts the energy management system that monitors and controls the dispatch of generation and demand to the FESS during testing;

- Back-to-Back Power Converter: A 540 kVA programmable B2B power converter interfaces the simulated power system model in the DRTS with the device under test (DUT), in this case the FESS;

- 1.2 MVA transformer: this transformer supplies power to the B2B converter but does not actually interface to the simulated power system;

- DUT: The device under test in this case is the GKN FESS, this flywheel has an energy storage capacity of $3.5 \mathrm{MJ}$ and a $360 \mathrm{~kW}$ power capability.

The DRTS simulation model has two components: (1) an electrical system model that represents the ship power system and (2) a control system model that controls the demand and generation within the power system

- MEPS: A simplified representation of a MEPS was used for this case study. A higher fidelity model can be implemented within the real time simulator however a simplified representative model was chosen for this test as: it meets the requirements for testing the FESS, simpli- fies the development and debugging process, and reduces the risk of instability in the closed loop system response. This ship model contains two AC sources representing the ship generators and an average value model of an ACDC converter. The converter interfaces the $\mathrm{AC}$ and $\mathrm{DC}$ networks of the ship power system. On the DC side of the ship, a Simulated Energy Magazine (SEM) incorporates a Lithium Ion battery and two programmable DC loads. The FESS is interface to the simulated DC network.

- Energy Management System: The energy management system (EMS) controls the load dispatch and generation supply considering the scenario that is being simulated. The generation supply includes the generators in the AC network (via the AC-DC converter), the flywheel, and the battery. The overarching control objective of the EMS is to limit the impact on the AC network while continuing to supply the loads. This is implemented by limiting the contribution of the AC/DC converter to an upper current setpoint, allowing it to supply a load or charge the FESS and battery up to this threshold. Any DC load demand beyond the limit of the converter current setpoint is met by discharging the FESS and BESS.

2) Step change characterisation tests: The objective of this part of testing is to evaluate and characterise the FESS behaviour at the PNDC, utilising the test configuration pre- 
TABLE II: Test Sequence

\begin{tabular}{cc}
\hline \hline Step Sequence (A) & Hold Duration (s) \\
\hline \hline$[0,-50,-200,-400,-200,-50,0,50,200,400,200,0]$ & $\begin{array}{c}2 \mathrm{~s} \\
(\text { each } \text { step })\end{array}$ \\
\hline \hline
\end{tabular}

TABLE III: Dropout test sequence

\begin{tabular}{cccc}
\hline \hline $\begin{array}{c}\text { Test } \\
\text { Stage }\end{array}$ & $\begin{array}{c}\text { Step Sequence } \\
(\mathrm{A})\end{array}$ & $\begin{array}{c}\text { Hold Duration } \\
(\mathrm{s})\end{array}$ & $\begin{array}{c}\text { Drop out } \\
\text { Duration (s) }\end{array}$ \\
\hline \hline 1 & {$[0,200,0,-200,0]$} & 4 & 0.001 \\
2 & {$[0,200,0,-200,0]$} & 4 & 0.01 \\
3 & {$[0,200,0,-200,0]$} & 4 & 0.1 \\
\hline \hline
\end{tabular}

sented in the previous section. In the first test a sequence of step changes in the FESS current reference was applied. The objective of this test is to inform on behaviour relating to delays in the FESS response and ramp rate limitations. The procedure for this first of the tests is listed in Table II. The associated setpoint (dotted line) and response is shown in Fig. $6 \mathrm{~b}$. The next test builds on the previous test by characterising how the FESS control responds to momentary changes in the FESS reference. These tests provide a constant reference to the FESS for charging or discharging. Once the FESS current is ramped up to the requested value and has reached steady state, the reference is momentarily stepped back to zero before being stepped back to the original reference level. The durations of these "dropout" events are varied in order to determine the minimum duration to which the FESS would respond. The sequence of drop out tests are explained in Table III and the responses are shown in Fig. 6c.

Both of these tests were used to tune the response of the EMS to compensate for the behaviour of the FESS. This meant implementing feed-forward control to compensate for the delay of the FESS in responding and also ensuring control signals are held for a minimum period so that the FESS control system has time to respond. Additional tests were conducted where the response of the FESS was coordinated with the BESS (i.e. the energy magazine), however the results will be reported in consequent publication.

3) Discussion: From the case study presented, it is evident that test beds play an important role in the acceleration of novel technology development and deployment. PNDC provides a testing platform close to the realistic expected operational environment of the developed technology for MEPS equipment to be experimentally evaluated. In this test platform no up-scaling or down-scaling of voltage or currents is required. This enables the appraisal of novel technologies to TRL 6.

\section{Systems Testing for Future Marine ELECTRICAL POWER SYSTEMS}

From the experimental evaluation methods of land based electrical power systems discussed in Section II and the adaptation of research infrastructures designed for land based electrical power systems experimental evaluation for MEPS testing (with its examples presented in Section III), this section discusses the future needs for systems testing of MEPS. Similar to land based power systems experimental evaluation, the characterization of novel components and controls in isolation is insufficient; where for rigorous evaluation the phase wise validation chain must be adopted. Combined CHIL and PHIL validation plays an important role that allows for a number of interactions to be investigated including: load balancing, power quality impact, protection system interactions, and response to operational scenarios. Without such testing the alternative is either to setup costly shore demonstrators or implement directly in the operational environment (with all the risks to operation that this entails). The testing reported in this paper involves two case studies where novel technology integration is explored. There are several ways this could be expanded beyond the testing reported in this paper and these are discussed below:

- The real world power system, i.e., the power system represented by the hardware of the test bed, could be expanded to include additional test hardware. This could involve additional loads (real or representative load banks) and/or additional alternative technology energy storage devices e.g. batteries or super capacitors. This adds realism to the system and would allow studies that explore the interaction between real hardware in the context of a MEPS.

- The notional power systems discussed in this paper could be expanded to represent higher fidelity ship power systems models. This would allow the operation of the novel components and controls to be explored in the context of specific MEPS platforms. The findings from such studies may indicate that the components or controls under investigation has a greater impact on some types of MEPS platform but not on others.

- Multiple PHIL interfaces could be implemented by adding additional points of common coupling. This would allow for more than one component to be connected at different locations within the simulated power system. This might be important when the impact on power transfer across the MEPS platform is to be tested. For example, when an energy storage device is supporting a load that is electrically distant or when the feasibility of zonal control is to be evaluated.

- Studies that consider specifically the impact of the control system design could indicate control systems that are more stable and reliable during different operational scenarios. Within this paper, intelligent controls were integrated within the MEPS to demonstrate the advantages they have to offer. Supporting intelligent controls requires increased observability within the network and adequate communications infrastructure for reliability.

\section{CONClusions}

In this paper, the systems testing of electrical power systems is discussed. Two smart grid enabled marine electrical 
power systems (MEPS) test beds developed with the research infrastructures available at University of Strathclyde have been presented. The value of these test beds for rapid proof of concept validations and component characterization have been demonstrated by means of two case studies. The complementarity of the two research infrastructures, Dynamic Power Systems Laboratory and Power Networks Demonstration Centre, is highlighted by means of a case study at each infrastructure, where the prior is utilized for appraisal of novel technologies, controls and architectures to technology readiness level (TRL) 5 and the later for validation at scale and under operational environment achieving TRL 7. Building upon the case studies presented and the land based power systems testing experiences of the two infrastructures, the future needs for systems level testing for MEPS is discussed. Future work includes the development of systems level testing methodology for MEPS with concrete examples of its applications.

\section{ACKNOWLEDGMENT}

This work in this paper has been supported by the RollsRoyce University Technology Centre. The work has also been in part supported by Directorate of Engineering and Safety (DE\&S) via Defence Science and Technology Laboratory (DSTL) Platform Systems Division. Any opinions, findings, and conclusions or recommendations expressed in this material are those of the authors and do not necessarily reflect those of the funding organizations.

\section{REFERENCES}

[1] T. Strasser, F. Pröstl Andrén, G. Lauss et al., "Towards holistic power distribution system validation and testing - an overview and discussion of different possibilities," e \& i Elektrotechnik und Informationstechnik, vol. 134, no. 1, pp. 71-77, Feb 2017.

[2] T. Strasser, M. Stifter, F. Andrn, and P. Palensky, "Co-simulation training platform for smart grids," IEEE Trans. on Power Syst., vol. 29, no. 4, pp. 1989-1997, July 2014.

[3] F. M. Albatsh, S. Mekhilef, S. Ahmad, and H. Mokhlis, "Fuzzy-logicbased upfe and laboratory prototype validation for dynamic power flow control in transmission lines," IEEE Trans. on Ind. Elect., vol. 64, no. 12, pp. 9538-9548, Dec 2017.

[4] X. Guillaud, M. O. Faruque, A. Teninge et al., "Applications of real-time simulation technologies in power and energy systems," IEEE Power and Energy Tech. Syst. Journal, vol. 2, no. 3, pp. 103-115, Sep. 2015.

[5] UK Power Networks, "Flexible Urban Networks Low Voltage Successful demonstrations of enhanced modes of operation of power electronics devices", (UK Power Networks, 2015), pp 1-70.

[6] J. Sanchez, D. Wetz, Q. Dong, and J. Heinzel, "Integration and study of hardware in the loop diesel generator with a hybrid energy storage module for naval applications," in 2017 IEEE Electric Ship Technologies Symposium (ESTS), Aug 2017, pp. 580-585.

[7] J. Langston, M. Steurer, K. Schoder, J. Borraccini, D. Dalessandro, T. Rumney, and T. Fikse, "Power hardware-in-the-loop simulation testing of a flywheel energy storage system for shipboard applications," in 2017 IEEE Electric Ship Technologies Symposium (ESTS), Aug 2017, pp. 305-311.

[8] S. Strank, X. Feng, A. Gattozzi, D. Wardell, S. Pish, J. Herbst, and R. Hebner, "Experimental test bed to de-risk the navy advanced development model," in 2017 IEEE Electric Ship Technologies Symposium (ESTS), Aug 2017, pp. 352-358.

[9] Sandia National Laboratories, "Test protocols for advances inverter interoperability functions", (Sandia Corporation, 2013), pp 1-22.

[10] E. Widl, P. Palensky, P. Siano, and C. Rehtanz, "Guest editorial modeling, simulation, and application of cyber-physical energy systems," IEEE Trans. on Ind. Informatics, vol. 10 (4), pp. 2244-2246, 112014.
[11] International Energy Agency, "Technology roadmap Smart Grids", (IEA, 2011), pp. 1-52

[12] M. Maniatopoulos, D. Lagos, P. Kotsampopoulos, and N. Hatziargyriou, "Combined control and power hardware in-the-loop simulation for testing smart grid control algorithms," IET Generation, Transmission Distribution, vol. 11, no. 12, pp. 3009-3018, 2017.

[13] M. Perron, E. Ghahremani, A. Heniche, I. Kamwa, C. Lafond, M. Racine, H. Akremi, P. Cadieux, S. Lebeau, and S. Landry, "Widearea voltage control system of flexible ac transmission system devices to prevent voltage collapse," IET Generation, Transmission Distribution, vol. 11, no. 18, pp. 4556-4564, 2017.

[14] J. Coser, A. S. Costa, and J. G. Rolim, "Metering scheme optimization with emphasis on ensuring bad-data processing capability," IEEE Transactions on Power Systems, vol. 21, no. 4, pp. 1903-1911, Nov 2006.

[15] J. Xu, C. Zhao, W. Liu, and C. Guo, "Accelerated model of modular multilevel converters in pscad/emtdc," IEEE Transactions on Power Delivery, vol. 28, no. 1, pp. 129-136, Jan 2013.

[16] J. Tant and J. Driesen, "On the numerical accuracy of electromagnetic transient simulation with power electronics," IEEE Transactions on Power Delivery, vol. 33, no. 5, pp. 2492-2501, Oct 2018.

[17] K. Johnstone, S. M. Blair, M. H. Syed, A. Emhemed, G. M. Burt, and T. I. Strasser, "Co-simulation approach using powerfactory and matlab/simulink to enable validation of distributed control concepts within future power systems," CIRED - Open Access Proceedings Journal, vol. 2017, no. 1, pp. 2192-2196, 2017.

[18] C. Steinbrink, S. Lehnhoff, S. Rohjans et al., "Simulation-based validation of smart grids - status quo and future research trends," in Industrial Applications of Holonic and Multi-Agent Systems. Cham: Springer International Publishing, 2017, pp. 171-185.

[19] J. Jeon, J. Kim, H. Kim, S. Kim, C. Cho, J. Kim, J. Ahn, and K. Nam, "Development of hardware in-the-loop simulation system for testing operation and control functions of microgrid," IEEE Transactions on Power Electronics, vol. 25, no. 12, pp. 2919-2929, Dec 2010.

[20] M. O. Omar Faruque and V. Dinavahi, "Hardware-in-the-loop simulation of power electronic systems using adaptive discretization," IEEE Transactions on Industrial Electronics, vol. 57, no. 4, pp. 1146-1158, April 2010.

[21] P. C. Kotsampopoulos, F. Lehfuss, G. F. Lauss, B. Bletterie, and N. D. Hatziargyriou, "The limitations of digital simulation and the advantages of phil testing in studying distributed generation provision of ancillary services," IEEE Transactions on Industrial Electronics, vol. 62, no. 9 , pp. 5502-5515, Sep. 2015.

[22] G. F. Lauss, M. O. Faruque, K. Schoder, C. Dufour, A. Viehweider, and J. Langston, "Characteristics and design of power hardware-in-theloop simulations for electrical power systems," IEEE Transactions on Industrial Electronics, vol. 63, no. 1, pp. 406-417, Jan 2016.

[23] M. Heder, "from nasa to eu: the evolution of the trl scale in public sector innovation," The Innovation Journal, pp. 1-23, Jan 2017.

[24] E. GuilloSansano, M. H. Syed, A. J. Roscoe, and G. M. Burt, "Initialization and synchronization of power hardware-in-the-loop simulations: A great britain network case study," Energies 2018, vol. 11, no. 5, p. 1087, 2018.

[25] S. M. Blair, N. Matheson, R. Munro, and C. Booth, "A new platform for validating real-time, large-scale wampac systems," in Protection, Automation and Control World Conference 2019, Glasgow, 2019, pp. $1-5$.

[26] T.-L. Nguyen, E. Guillo-Sansano, M. H. Syed, V.-H. Nguyen, S. M. Blair, L. Reguera, Q.-T. Tran, R. Caire, G. M. Burt, C. Gavriluta, and N.A. Luu, "Multi-agent system with plug and play feature for distributed secondary control in microgridcontroller and power hardware-in-theloop implementation." Energies 2018, vol. 11, no. 12, p. 3253, 2018.

[27] M. Stubs, P. Dambrauskas, M. H. Syed, K. Koster, H. Federrath, G. M. Burt, and T. I. Strasser, "calable power system communications emulation with opc ua," CIRED - Open Access Proceedings Journal, vol. 2019, no. 1, pp. 1-5, 2019.

[28] E. Guillo-Sansano, A. J. Roscoe, and G. M. Burt, "Harmonic-byharmonic time delay compensation method for phil simulation of low impedance power systems," in 2015 International Symposium on Smart Electric Distribution Systems and Technologies (EDST), Sep. 2015, pp. 560-565. 